\title{
Benzo- and naphthoimidazoxadiazolediene, naphthobisthiazole as well as naphthothiazine derivatives from 1-acylthiosemicarbazides
}

\author{
Alaa A. Hassan, Aboul-Fetouh E. Mourad, and Ashraf H. Abou-Zied \\ Chemistry Department, Faculty of Science, El-Minia University, El-Minia, A. R. Egypt \\ E-mail: alaahassan2001@yahoo.com
}

\begin{abstract}
1-Acylthiosemicarbazides 1a-d reacted with 2,3-dichloro-5,6-dicyano-1,4-benzoquinone (2a), 2,3,5,6-tetrachloro-1,4-benzoquinone (2b), 2,3-dichloro-1,4-naphthoquinone (3a) and 2,3dicyano-1,4-naphthoquinone (3b) in ethyl acetate with admission of air to form benzo- and naphtho-imidazoxadiazoles $(\mathbf{5}, \mathbf{6}, \mathbf{1 1})$, naphthobisthiazoles (12a-d), naphthothiadiazines (13a-d) as well as 2,3,7,8-tetrachlorothianthrene-1,4,6,9-tetraone (7). Rationales for the observed conversions are presented.
\end{abstract}

Keywords: Benzo- and naphthoquinones, Cyclocondensation, Fused heterocyclic compounds

\section{Introduction}

The chemistry of quinones is of considerable interest since this class of compounds includes many natural products and numerous important synthetic products ${ }^{1,2}$. Addition of nitrogen nucleophiles to benzo- and naphthoquinones represents a common synthetic route to many fused heterocyclic rings which have been used as synthetic intermediates in medicinal chemistry ${ }^{3-6}$ and for dyestuffs ${ }^{7-16}$.

2,3,5,6-Tetrachloro-1,4-benzoquinone (2b) and 2,3-dichloro-1,4-naphthoquinone (3a) reacted with $N^{1}, N^{2}$-diarylamidines to give benzimidazole and indole derivatives ${ }^{17,18}$. A series of benzo- and naphthothiazolediones have been synthesized by reaction of $N$-substituted thioureas with $\mathbf{2 a}, \mathbf{2} \mathbf{b}$ and $\mathbf{3} \mathbf{a}^{19}$. Indazole, thiadiazine and naphothiadiazine derivatives were isolated from the reaction of thiosemicarbazide with $\mathbf{2} \mathbf{b}$ and $\mathbf{3 a}^{10}$. The reaction of $N, N^{\prime}$-disubstituted hydrazinecarbothioamides with $\mathbf{2} \mathbf{b}$ and $\mathbf{3 a}$ afforded thiadiazole and thiadiazine derivatives ${ }^{20}$. In contrast, quinoxaline and thiadiazepane derivatives were obtained from the reaction of substituted thioureidoethylthioureas with $\mathbf{2} \mathbf{b}^{20}$.

Recently, we have reported that 4-substituted thiosemicarbazides reacted with $\mathbf{2 a}, \mathbf{2 b}$ and $\mathbf{3 a}$ in ethyl acetate with admission of air to form derivatives of 1,5,2,3-oxathiadiazole, indazole, thiadiazine-6-one, 1,3,4-thiadiazaphenanthrenone and naphtho[1,2-e $: 4,3-e$ ]bis[1,3,4]- 
thiadiazine $^{21}$. This unique reactivity has no precedence and warrants further investigation. Therefore, we undertook to prepare electron poorer examples such as 1-acylthiosemicarbazides $\mathbf{1 a - d}$, and to investigate their behaviour towards benzo- as well as naphthoquinones $\mathbf{2 a , b}$ and 3a,b (Fig. 1).<smiles>[R]C(=O)NNC(N)=S</smiles>

1a-d

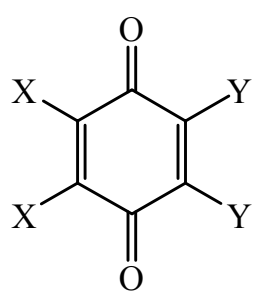

2: $\mathbf{a}, \mathrm{X}=\mathrm{CN}, \mathrm{Y}=\mathrm{Cl}(\mathbf{D D Q})$

b, $\mathrm{X}=\mathrm{Y}=\mathrm{Cl}(\mathbf{C H L})$<smiles>[X]C1=C([X])C(=O)c2ccccc2C1=O</smiles>

3: $\mathbf{a}, \mathrm{X}=\mathrm{Cl}(\mathbf{D C H N Q})$

b, $\mathrm{X}=\mathrm{CN}(\mathbf{D C N Q})$

\begin{tabular}{c|c|c|c|c}
$\mathbf{1}$ & $\mathbf{a}$ & $\mathbf{b}$ & $\mathbf{c}$ & $\mathbf{d}$ \\
\hline $\mathrm{R}$ & $\mathrm{CH}_{3}$ & $\mathrm{C}_{6} \mathrm{H}_{5}$ & $4-\mathrm{HO}-\mathrm{C}_{6} \mathrm{H}_{4}$ & $4-\mathrm{Br}_{-} \mathrm{C}_{6} \mathrm{H}_{4}-\mathrm{CH}_{2}$
\end{tabular}

Figure 1

\section{Results and Discussion}

Mixing of two-fold molar amounts of $\mathbf{2 a}$ with one mole each of the donors 1a-d in ethyl acetate with admission of air gives a blue colour $\left(\lambda_{\max }=573-591 \mathrm{~nm}\right)$. This colour changes gradually to brown with the formation of a solid product. This behaviour is explained as being due to initial formation of an unstable charge-transfer complex (CTC) followed by a chemical reaction which yields substituted benzimidazoxadiazole 5a-d via the reaction of dihydrobenzoquinone $\left(\mathbf{2 a}-\mathrm{H}_{2}\right)$ with 4 and elimination one molecule of $\mathrm{HCN}$ and another of $\mathrm{H}_{2} \mathrm{O}$ (Scheme 1). The structures of the well known compounds 4a-c were confirmed on the basis of spectral data and mixed melting points. The structural assignments for the benzimidazoxadiazole derivatives 5a-d are based on the following spectral data: the IR spectrum of 5a showed characteristic absorption for the hydroxyl group at $v 3440 \mathrm{~cm}^{-1}$ and at $2220 \mathrm{~cm}^{-1}$ for the cyano group. The ${ }^{1} \mathrm{H}-\mathrm{NMR}$ spectrum showed a broad signal at $9.53 \mathrm{ppm}$ due to the $\mathrm{OH}$ in addition to the methyl group at $2.33 \mathrm{ppm}$. The decoupled ${ }^{13} \mathrm{C}$-NMR spectrum showed signals at $\delta 164.82,156.22$ and 150.71 for C-2, C-9a and C-8a, respectively. Also, the ${ }^{13} \mathrm{C}-\mathrm{NMR}$ clearly indicates the presence of one cyano group at 118.77 ppm beside the aromatic carbons.

The molecular formulae for 5a-d (Scheme 1) are supported by elemental analyses and mass spectra, which gave the expected molecular ion peaks. The semi-micropreparative scale reaction of 1a with 2a gave 5a, as established from the comparison of its IR spectrum and mp with those 
of an authentic sample. In addition, small quantities of numerous coloured, unidentifiable byproducts were observed.

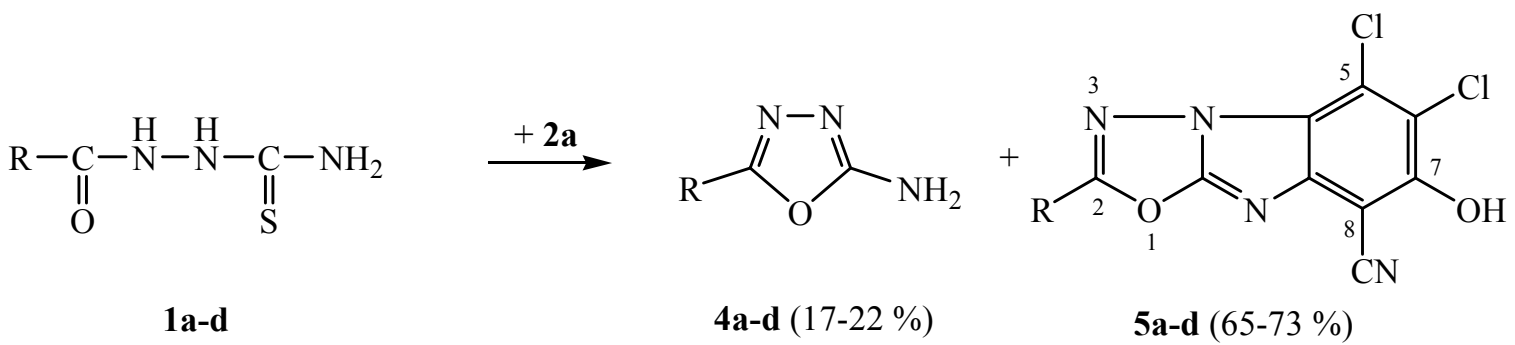

\begin{tabular}{c|c|c|c|c}
$\mathbf{1 , 4 , 5}$ & $\mathbf{a}$ & $\mathbf{b}$ & $\mathbf{c}$ & $\mathbf{d}$ \\
\hline $\mathrm{R}$ & $\mathrm{CH}_{3}$ & $\mathrm{C}_{6} \mathrm{H}_{5}$ & $4-\mathrm{HO}-\mathrm{C}_{6} \mathrm{H}_{4}$ & $4-\mathrm{Br}_{-} \mathrm{C}_{6} \mathrm{H}_{4}-\mathrm{CH}_{2}$
\end{tabular}

\section{Scheme 1}

On the other hand, mixing of a two fold molar excess of $\mathbf{2 b}$ with one mole of 1acylthiosemicarbazides 1a-d leads to the formation of an initial CTC $\left(\lambda_{\max }=506-518 \mathrm{~nm}\right)$ followed by formation (complete after three days) of the products, benzimidazoxadiazolediones 6a-d, oxadiazoles 4a-d and 2,3,7,8-tetrachlorothianthrene-1,4,6,9- tetraone 7 (Scheme 2).

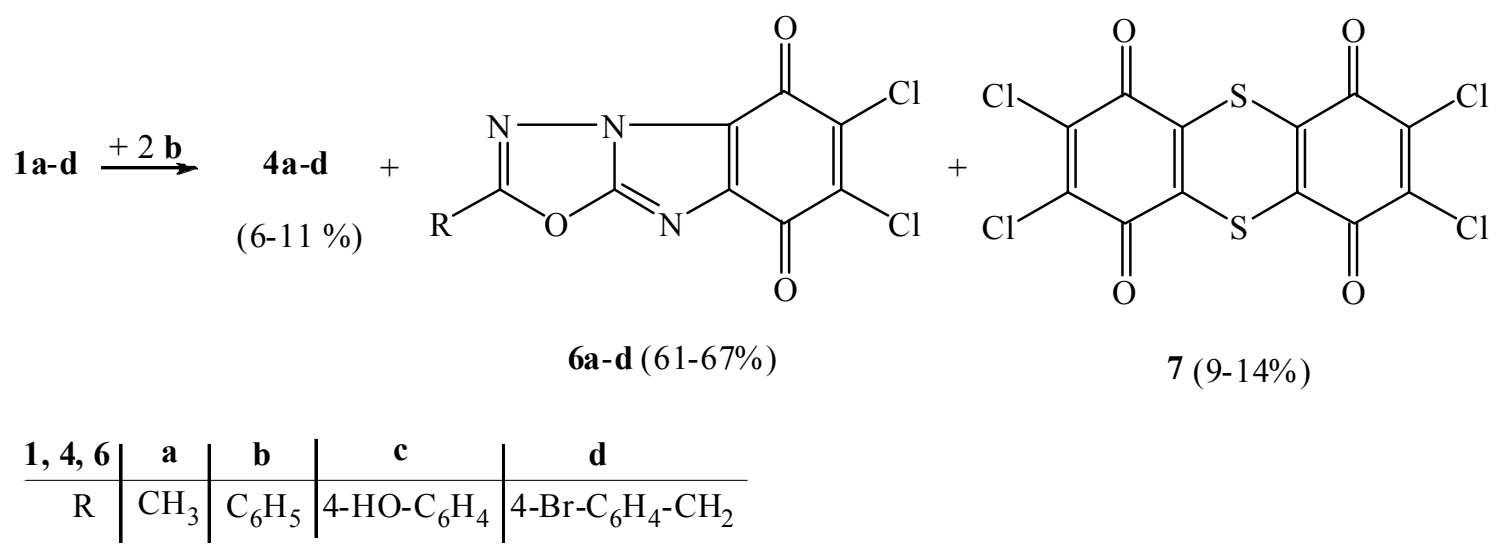

\section{Scheme 2}

The IR spectrum of $\mathbf{6 b}$ showed a sharp band at $1695 \mathrm{~cm}^{-1}$ for the carbonyl group of the quinone system. The ${ }^{1} \mathrm{H}-\mathrm{NMR}$ spectrum revealed a multiplet at 7.19-7.66 ppm, which is characteristic of phenyl protons. The ${ }^{13} \mathrm{C}$-NMR spectrum showed the characteristic absorption signals of the carbonyl carbon atoms of $\mathbf{2 b}$ at 170.72 and $171.83^{22}$. Other signals were observed in the ${ }^{13} \mathrm{C}-\mathrm{NMR}$ of $\mathbf{6 b}$, clearly indicating the presence of $\mathrm{C}=\mathrm{N}, \mathrm{N}=\mathrm{C}-\mathrm{O}, \mathrm{Cl}-\mathrm{C}=\mathrm{C}=\mathrm{Cl}$ groups 
(experimental part). The formation of $\mathbf{6 b}$ was further confirmed by mass spectrometry. Besides the molecular ion at 331/335, the characteristic fragment ion patterns of substituted dichloro compounds were observed ${ }^{23}$.

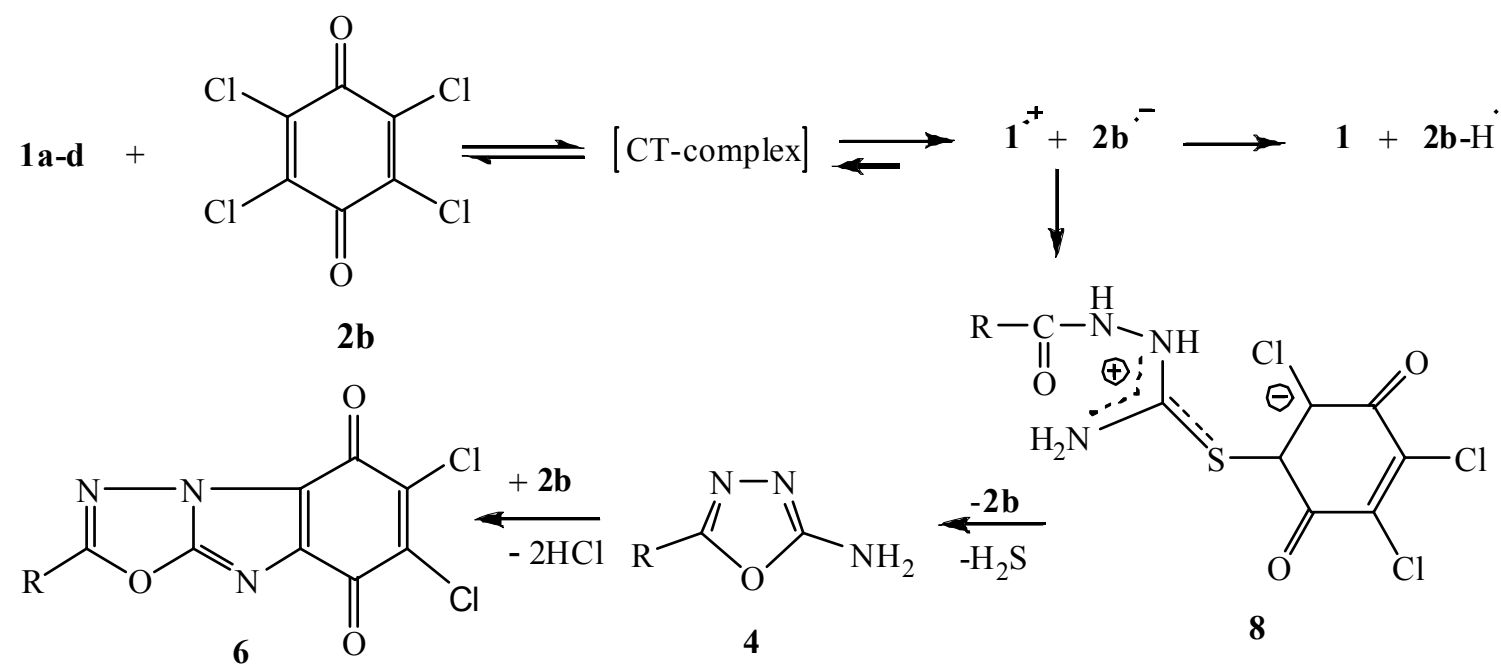<smiles>[R]C(=NC1=NNC(N)=NC1SC1=C(Cl)C(=O)C(Cl)=C(Cl)C1=O)OCC</smiles><smiles>O=c1c(Cl)c(Cl)c(=O)c2sc3c(=O)c(Cl)c(Cl)c(=O)c3sc12</smiles>

7

\section{Scheme 3}

Formation of products 4, 6 and 7 may be rationalized by the mechanism shown in Scheme 3. An unstable CTC is formed followed by the formation of radical $\mathbf{1}^{\cdot}$ and $\mathbf{2 b}-\mathrm{H}^{\cdot}$.

Two routes can be suggested for the formation of compounds 4, 6 and 7 . The first one is the cyclization of 1a-d and formation of the oxadiazoles 4a-d during intramolecular nucleophilic attacks on the thiocarbonyl group. After cyclization, $\mathbf{2 b}$ is released with the liberation of $\mathrm{H}_{2} \mathrm{~S}$ (Scheme 3). Recombination of $\mathbf{4}$ and $\mathbf{2 b}$ with elimination of two molecules of $\mathrm{HCl}$ would afford 
the benzimidazoxodiazolediones 6a-d. The second possible route is the elimination of one molecule of $\mathrm{HCl}$ from $(\mathbf{1}+\mathbf{2 b}-\mathbf{H})$ to give the intermediate $\mathbf{9}$. Nucleophilic attack by the $\mathrm{OH}$ group on $\mathrm{C}=\mathrm{N}$ and detachment of the HS-moiety would afford the intermediate $\mathbf{1 0}$ along with oxadiazoles 4a-d. Then, the tetrachlorothianthrenetetraone 7 could be formed via the reaction of two molecules of $\mathbf{1 0}$ with the elimination of two molecules of $\mathrm{HCl}$ (Scheme 3).

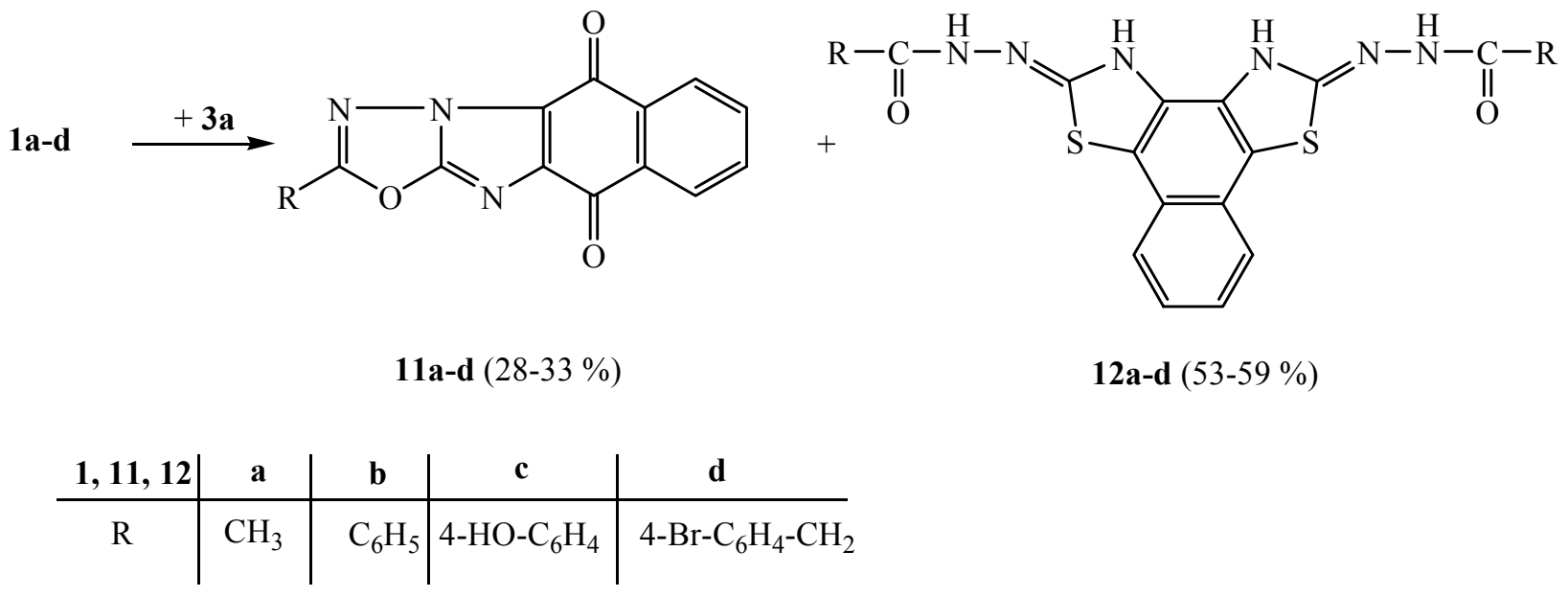

\section{Scheme 4}

By mixing equimolar amounts of 1-acylthiosemicarbazides 1a-d and 3a in ethyl acetate the colour of the reaction mixture remains unchanged. Obviously, there is no donor-acceptor interaction between these two molecules, which is mainly due to the low electron affinity of $3 \mathbf{a}$ compared with $\mathbf{2} \mathbf{b}^{24}$. Heating of this mixture for 5 hours and chromatographic separation of the residue after concentration gave numerous coloured zones, from which naphthoimidazoxadiazoles 11a-d and naphthobisthiazoles 12a-d could be isolated (Scheme 4).

The structures of 11a-d were delineated from their spectroscopic properties and gross compositions. The major products $\mathbf{1 2 a - d}$ were found to be formed from one molecule of $\mathbf{3 a}-\mathrm{H}_{2}$ and two molecules of 1a-d by loss of two molecules of $\mathrm{H}_{2} \mathrm{O}$ and $\mathrm{HCl}$.

The IR spectrum of 12d showed absorption characteristic of $\mathrm{NH}$ groups at $3385,3225 \mathrm{~cm}^{-1}$ and a strong carbonyl group absorption at $1670 \mathrm{~cm}^{-1}$. The ${ }^{1} \mathrm{H}-\mathrm{NMR}$ spectrum of $\mathbf{1 2 d}$ clearly indicates the presence of two different broad signals centered at 10.65 and $11.17 \mathrm{ppm}$ due to thiazole-NH and amide-NH, respectively. In addition, the benzylic- $\mathrm{CH}_{2}$ as well as aromatic protons were observed (see experimental part). The ${ }^{13} \mathrm{C}-\mathrm{NMR}$ of $\mathbf{1 2 d}$ showed a carbonyl signal at $\delta_{\mathrm{C}}=171.48$ corresponding to the amide group. Also, the ${ }^{13} \mathrm{C}-\mathrm{NMR}$ clearly indicates the presence of signals at 52.28 and 163.26 due to benzylic- $\mathrm{CH}_{2}$ and thiazole- $\mathrm{C}_{2}$, respectively. The elemental analysis of $\mathbf{1 2 d}$ suggested a gross formula $\mathrm{C}_{28} \mathrm{H}_{20} \mathrm{Br}_{2} \mathrm{~N}_{6} \mathrm{O}_{2} \mathrm{~S}_{2}$ and this was confirmed by the mass spectrum, which exhibited the molecular ion at $\mathrm{m} / \mathrm{z}$ 698/694 (17\%). It should be noted also that the mass spectra of compounds 12a-d show the loss of an acyl group from the molecular ions. 
In contrast to the situation with $\mathbf{3 a}$, on addition of $\mathbf{1 a - d}$ to $\mathbf{3 b}$, the initial formation of CT complexes $\left(\lambda_{\max }=523-532 \mathrm{~nm}\right)$ is followed by the formation of naphthothiazine derivatives 13a$\mathbf{d}$ in addition to oxadiazoles 4a-d (Scheme 5).

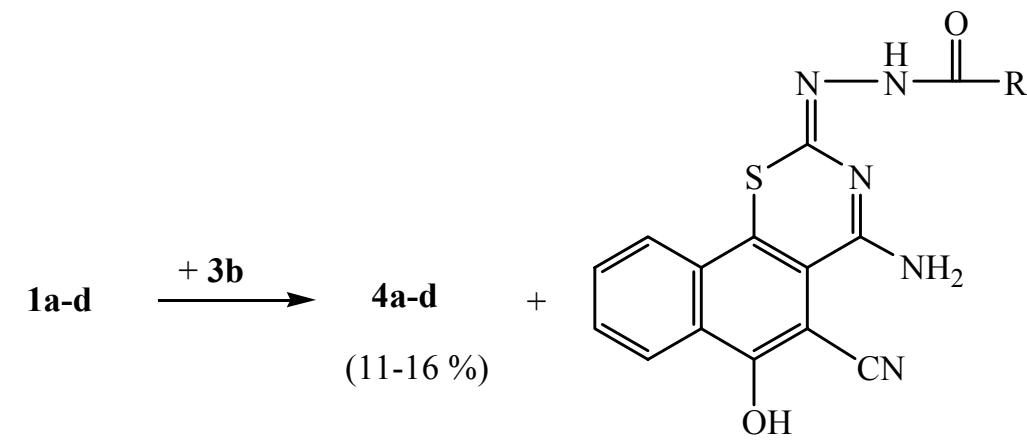

13a-d $(65-72 \%)$

\begin{tabular}{c|c|c|c|c}
$\mathbf{1 , 4 , 1 3}$ & $\mathbf{a}$ & $\mathbf{b}$ & $\mathbf{c}$ & $\mathbf{d}$ \\
\hline $\mathrm{R}$ & $\mathrm{CH}_{3}$ & $\mathrm{C}_{6} \mathrm{H}_{5}$ & $4-\mathrm{HO}-\mathrm{C}_{6} \mathrm{H}_{4}$ & $4-\mathrm{Br}_{-} \mathrm{C}_{6} \mathrm{H}_{4}-\mathrm{CH}_{2}$
\end{tabular}

\section{Scheme 5}

For compound $\mathbf{1 3 b}$, the gross formula $\mathrm{C}_{20} \mathrm{H}_{13} \mathrm{~N}_{5} \mathrm{O}_{2} \mathrm{~S}$ is supported by mass spectroscopy, which clearly demonstrates the loss of a benzoyl group. The ${ }^{13} \mathrm{C}$ NMR spectrum reveals the absence of the $\mathrm{C}=\mathrm{S}$ signal and the presence of an amide $\mathrm{C}=\mathrm{O}$ signal (171.56) and only one $\mathrm{CN}$ resonance $(118.11 \mathrm{ppm})$. In addition to an $\mathrm{OH}$ group, both a $\mathrm{NH}_{2}\left(\delta_{\mathrm{H}}=7.12 \mathrm{ppm}\right)$ and a low field amide-NH $\left(\delta_{\mathrm{H}}=11.15 \mathrm{ppm}\right)$ are present. The IR spectrum of $\mathbf{1 3 b}$ showed bands at 3445, 33703250,2220 and $1675 \mathrm{~cm}^{-1}$ due to $\mathrm{OH},\left(\mathrm{NH}\right.$ and $\left.\mathrm{NH}_{2}\right), \mathrm{CN}$ and amide $\mathrm{C}=\mathrm{O}$ groups, respectively.

\section{Conclusions}

Novel and interesting structures are presented here from the reactions between the electron donor 1-acylthiosemicarbazides 1a-d and electron acceptors; benzo- as well as naphthoquinones 2a,b and 3a,b. In a fairly complex, multistep process, three interesting kinds of fused heterocyclic compounds (benzo- and naphthoimidazoxadiazoles, naphthobisthiazole and naphthothiazine derivatives) are formed, in addition to the oxadiazole ring. Thus, benzo- and naphthoquinones may act either as mediators or as building blocks in heterocyclization of acylthiosemicarbazides. The results reported also supplement the chemistry of nucleophilic substitution of halogenated $p$ quinones, which continues to be of interest for the synthesis of many heterocycles. 


\section{Experimental Section}

General Procedures: The uncorrected melting points were determined on a Gallenkamp melting point apparatus, IR spectra were recorded using $\mathrm{KBr}$ disks on Shimadzu 408 or Bruker Vector 22 FT-IR instruments. ${ }^{1} \mathrm{H} 300 \mathrm{MHz}$ and ${ }^{13} \mathrm{C}-\mathrm{NMR} 75 \mathrm{MHz}$ spectra were recorded on a Bruker WM300 instrument, $500 \mathrm{MHz}{ }^{1} \mathrm{H}$ and $125 \mathrm{MHz}{ }^{13} \mathrm{C}-\mathrm{NMR}$ spectra on a Bruker DRX 500 spectrometer. Chemical shifts are expresses as $\delta[\mathrm{ppm}]$ with reference to tetramethylsilane as an internal standard, $\mathrm{s}=$ singlet $\mathrm{d}=$ doublet, $\mathrm{m}=$ multiplet. The ${ }^{13} \mathrm{C}$ signals were assigned on the basis of DEPT 135/90 spectra. The mass spectra (70 ev, electron impact mode) were recorded on an AMD 604 instrument. The UV-VIS spectra were recorded on a Perkin-Elmer Lambda 2 spectrophotometer. Combustion analysis was carried out at the Microanalytical center, Cairo University, Egypt. Preparative layer chromatography (plc) was carried out using air dried 1.0 $\mathrm{mm}$ thick layers of a slurry of silica gel $\left(\right.$ Merck $\left.\mathrm{PF}_{254}\right)$ applied on $48 \mathrm{~cm}$ wide and $20 \mathrm{~cm}$ high glass plates using cyclohexane/ ethyl acetate as developing solvent. Zones were detected by their colour or by quenching of indicator fluorescence upon exposure to $254 \mathrm{~nm}$ light and extracted out with acetone.

Materials: 1-Acylthiosemicarbazides 1a-c were prepared according to the literature ${ }^{25-27}$. The ${ }^{1} \mathrm{H}-\mathrm{NMR}$ spectral data of 1-acetylthiosemicarbazide (1a) ${ }^{25}, 1$-benzoylthiosemicarbazide $(\mathbf{1 b})^{26}$, and 1-(4-hydroxyphenyl)thiosemicarbazide (1c) ${ }^{27}$ were in full accord with the published data.

1-(4-Bromophenylaceto)thiosemicarbazide (1d). To a stirred solution of thiosemicarbazide $(0.91 \mathrm{~g}, 10 \mathrm{mmol})$ in $50 \mathrm{ml}$ dry acetone, $p$-bromophenylactic acid $(2.15 \mathrm{~g}, 10 \mathrm{mmol})$ was added and the mixture was refluxed for 3 hours. A white precipitate was formed and recrystallized from ethanol to give colourless crystals $(2.84 \mathrm{~g}, 85 \%), \mathrm{mp}=96-98{ }^{\circ} \mathrm{C}$.

2,3-Dichloro-5,6-dicyano-1,4-benzoquinone (2a, Aldrich) was recrystallized from benzene/chloroform (2:3). 2,3,5,6-Tetrachloro-1,4-benzoquinone (2b, Aldrich) was recrystallized from benzene before use. 2,3-Dicyano-1,4-naphthoquinone (3b) was prepared from 2,3-dichloro-1,4naphthoquinone (3a) according to $\mathrm{Badni}^{28}$ and recrystallized from dichloromethane.

\section{Reaction of 1-acylthiosemicarbazides 1a-d with 2a}

To a solution of $454 \mathrm{mg}$ of 2a (2 mmoles) in $20 \mathrm{ml}$ of dry ethyl acetate, were added the acylthiosemicarbazides 1a-d $(1 \mathrm{mmol})$ in $15 \mathrm{ml}$ of dry ethyl acetate dropwise with stirring at room temperature. Thereafter, the mixture was stirred for $24 \mathrm{~h}$, filtered, and the precipitate was washed with a small amount of cold ethyl acetate. The filtrate was concentrated and the residue chromatographed on thin-layer plates (silica gel $\mathrm{Pf}_{254}$ ) using cyclohexane/ethyl acetate (5:1) to give only one zone containing the oxadiazole derivatives 4a-d. Recrystallization of the isolated products from suitable solvents afforded the pure compounds 4a-d and 5a-d.

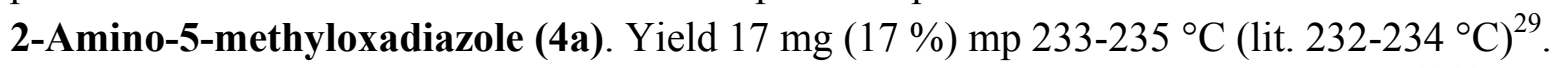
2-Amino-5-phenyloxadiazole (4b). Yield $19 \mathrm{mg}(19 \%) \mathrm{mp} 252-54{ }^{\circ} \mathrm{C}\left(\text { lit. } 250{ }^{\circ} \mathrm{C}\right)^{30,31}$. 
2-Amino-5-(4-hydroxyphenyl))oxadiazole (4c). Yield $22 \mathrm{mg}(19 \%) \mathrm{mp} \mathrm{286-288}{ }^{\circ} \mathrm{C}$ (lit. 288$\left.290{ }^{\circ} \mathrm{C}\right)^{30,31}$.

2-Amino-5-(4-bromobenzyl))oxadiazole (4d). Colourless crystals (21 mg, $21 \%$ ), mp 185-187 ${ }^{\circ} \mathrm{C}$ (ethanol). IR (KBr): v $3410\left(\mathrm{NH}_{2}\right), 1630(\mathrm{C}=\mathrm{N}), 1595$ (aryl), $1085(\mathrm{C}-\mathrm{O}-\mathrm{C}) \mathrm{cm}^{-1} .{ }^{1} \mathrm{H}-\mathrm{NMR}$ $\left(\mathrm{DMSO}_{6}\right.$ ): $\delta 4.21\left(\mathrm{~s}, 2 \mathrm{H}, \mathrm{CH}_{2}\right), 6.88$ (br, 2H, $\left.\mathrm{NH}_{2}\right), 7.14-7.69$ (m, 4H, aryl-H). MS m/z (\%): 255/253 ( $\mathrm{M}^{+}, 33$ ), 211 (21), 131 (36), 90 (87), 77 (100). Anal. Calcd. for $\mathrm{C}_{9} \mathrm{H}_{8} \mathrm{~N}_{3} \mathrm{BrO}$ : C, 42.54; H, 3.17; N, 16.54. Found: C, 42.78; H, 2.98; N, 16.29.

2-Methyl-5,6-dichloro-8-cyano-7-hydroxy[4,5]benzimidazo[2,1-b][1,3,4]oxadiazole

(5a). Brown crystals (193 mg, 68 \%), mp 135-137 ${ }^{\circ} \mathrm{C}$ (methanol). IR (KBr): v $3440(\mathrm{OH}), 2220(\mathrm{CN})$, $1625(\mathrm{C}=\mathrm{N}), 1600$ (aryl), $1090(\mathrm{C}-\mathrm{O}-\mathrm{C}) \mathrm{cm}^{-1} .{ }^{1} \mathrm{H}-\mathrm{NMR}\left(\mathrm{DMSO}_{6}\right): \delta 2.33\left(\mathrm{~s}, 3 \mathrm{H}, \mathrm{CH}_{3}\right), 9.53$ (br, 2H, OH). ${ }^{13} \mathrm{C}-\mathrm{NMR}$ (DMSO-d6): $\delta 16.68\left(\mathrm{CH}_{3}\right), 118.77(\mathrm{CN}), 121.44,121.88(\mathrm{C}-5, \mathrm{C}-6)$, 142.44 (C-8), 150.22 (C-4a), 150.71 (C-8a), 152.98 (C-7), 156.22 (C-9a), 164.82 (C-2). MS m/z (\%): 286/282 (M+, 18), 239 (22), 169 (29), 139 (12), 113 (45), 43 (100). Anal. Calcd. for $\mathrm{C}_{10} \mathrm{H}_{4} \mathrm{Cl}_{2} \mathrm{~N}_{4} \mathrm{O}_{2}: \mathrm{C}, 42.43 ; \mathrm{H}, 1.42 ; \mathrm{N}, 19.79 ; \mathrm{Cl}, 25.05$. Found: C, 43.62; H, 1.67; N, 19.57; Cl, 25.17 .

\section{2-Phenyl-5,6-dichloro-8-cyano-7-hydroxy[4,5]benzimidazo[2,1-b][1,3,4] oxadiazole}

(5b). Brown crystals $\left(252 \mathrm{mg}, 73 \%\right.$ ), mp 206-208 ${ }^{\circ} \mathrm{C}$ (acetonitrile). IR (KBr): v 3430 (OH), 2215 $(\mathrm{CN}), 1610(\mathrm{C}=\mathrm{N}), 1590$ (aryl), $1080(\mathrm{C}-\mathrm{O}-\mathrm{C}) \mathrm{cm}^{-1} .{ }^{1} \mathrm{H}-\mathrm{NMR}\left(\mathrm{DMSO}-\mathrm{d}_{6}\right): \delta$ 7.18-7.55 (m, 5H, aryl), 9.49 (br, 1H, OH). ${ }^{13} \mathrm{C}-\mathrm{NMR}$ (DMSO-d6): $\delta 118.74(\mathrm{CN}), 121.64,122.18$ (C-5, C-6), 127.66, 128.86, 129.38 (aryl-CH), 131.12 (aryl-C), 141.96 (C-8), 150.67 (C-4a), 150.88 (C-8a), 153.11 (C-7), 156.18 (C-9a), 164.88 (C-2). MS m/z (\%): 348/344 (M+16), 239 (29), 169 (18), 139 (22), 105 (100), 65 (33). Anal. Calcd. for $\mathrm{C}_{15} \mathrm{H}_{6} \mathrm{Cl}_{2} \mathrm{~N}_{4} \mathrm{O}_{2}$ : C, 52.20; H, 1.75; N, 16.23; Cl, 20.54. Found: C, 52.38; H, 1.91; N, 16.05; Cl, 20.62.

2-(4-Hydroxyphenyl)-5,6-dichloro-8-cyano-7-hydroxy [4,5]benzimidazo[2,1-b][1,3,4]oxadiazole (5c). Brown crystals (253 mg, $70 \%$ ), mp 155-157 ${ }^{\circ} \mathrm{C}$ (acetonitrile). IR (KBr): $v 3450$ $(\mathrm{OH}), 2220(\mathrm{CN}), 1625(\mathrm{C}=\mathrm{N}), 1600$ (aryl), $1090(\mathrm{C}-\mathrm{O}-\mathrm{C}) \mathrm{cm}^{-1} .{ }^{1} \mathrm{H}-\mathrm{NMR}\left(\mathrm{DMSO}-\mathrm{d}_{6}\right): \delta$ 6.96$7.4\left(\mathrm{~m}, 4 \mathrm{H}\right.$, aryl), 9.38 (br, 1H, OH), 9.52 (br, 1H, OH). ${ }^{13} \mathrm{C}-\mathrm{NMR}$ (DMSO-d6): $\delta 118.69(\mathrm{CN})$, 121.53, 122.10 (C-5, C-6), 126.12, 129.16 (aryl-CH), 130.08, 142.12 (C-8), 150.52 (C-4a), 151.10 (C-8a), 153.35 (C-7), 156.53 (aryl-C), 165.02 (C-2). MS m/z (\%): 364/360 (M+, 21), 239 (32), 169 (15), 121 (96), 105 (88), 93 (100), 77 (82), 65 (44). Anal. Calcd. for $\mathrm{C}_{15} \mathrm{H}_{6} \mathrm{Cl}_{2} \mathrm{~N}_{4} \mathrm{O}_{3}$ : C, 49.89; H, 1.67; N, 15.51; Cl, 19.63. Found: C, 50.06; H, 1.45; N, 15.73; Cl, 19.44.

2(4-Bromobenzyl)-5,6-dichloro-8-cyano-7-hydroxy[4,5]benzimidazo[2,1-b][1,3,4] oxadiazole (5d). Brown crystals (285 mg, $65 \%$ ), mp 220-222 ${ }^{\circ} \mathrm{C}$ (ethanol). IR (KBr): v 3435 (OH), 2220 $(\mathrm{CN}), 1620(\mathrm{C}=\mathrm{N}), 1595$ (aryl), $1086(\mathrm{C}-\mathrm{O}-\mathrm{C}) \mathrm{cm}^{-1} .{ }^{1} \mathrm{H}-\mathrm{NMR}\left(\mathrm{DMSO}-\mathrm{d}_{6}\right): \delta 4.73\left(\mathrm{~s}, 2 \mathrm{H}, \mathrm{CH}_{2}\right)$, 6.98-7.43 (m, 4H, aryl), 9.48(br, 1H, OH). ${ }^{13} \mathrm{C}-\mathrm{NMR}$ (DMSO-d6): $\delta 52.71\left(\mathrm{CH}_{2}\right), 118.69(\mathrm{CN})$, 121.62, 121.98 (C-5, C-6), 126.66 (aryl-C),128.33, 129.64 (aryl-CH),134.32 (aryl-C), 142.11 (C8), 150.72 (C-4a), 150.86 (C-8a), 153.14 (C-7), 156.14 (C-9a), 164.76 (C-2). MS m/z (\%): 442/436 (M+2 22), 356 (29), 239 (6), 189 (77), 142 (15), 91 (88), 77 (100), 65 (54). Anal. Calcd. for $\mathrm{C}_{16} \mathrm{H}_{7} \mathrm{BrCl}_{2} \mathrm{~N}_{4} \mathrm{O}_{2}$ : C, 43.87; H, 1.61; N, 12.79; Cl, 16.19. Found: C, 44.02; H, 1.54; N, 12.93; $\mathrm{Cl}, 16.03$. 


\section{Reaction of 1-acylthiosemicarbazides $1 \mathrm{a}-\mathrm{d}$ with $\mathbf{2 b}$}

To a stirred solution of $492 \mathrm{mg}$ ( 2 mmmols) of $\mathbf{2 b}$ in $30 \mathrm{ml}$ of dry ethyl acetate, were added acylthiosemicarbazides 1a-d $(1 \mathrm{mmol})$ in $15 \mathrm{ml}$ dry ethyl acetate dropwise at room temperature. The colour of the reaction mixture changed gradually from reddish brown to pale blue. The mixture was stirred for another $72 \mathrm{~h}$ and then filtered off. The blue precipitate which contained compound $7^{20}$ was washed with cold ethyl acetate. The filtrate was concentrated and the residue was then separated by preparative layer chromatography (plc) using a suitable eluent (cyclohexane/ ethyl acetate, 5:1 for the reaction of $\mathbf{2 b}$ with $\mathbf{1 a}$ and $\mathbf{1 d}$; 3:1 for the reaction of $\mathbf{2 b}$ with 1b and 1c) to give numerous coloured zones, two of which (with high intensity) were removed and extracted. The faster migrating one, $\mathrm{R}_{\mathrm{f}}=0.146$, contained the oxadiazoles 4a-d, and the second zone, $\mathrm{R}_{\mathrm{f}}=0.096$ (characterized by its green colour) contained benzimidazoxadiazolediones 6a-d. Extraction of the zones with acetone, and concentration, gave a residue which was rechromatographed to separate the pure compounds.

2-Methyl-6,7-dichloro-benzo[4,5]imidazo[2,1-b][1,3,4] $0 x$ adiazole-5,8-dione (6a). Pale green crystals (171 mg, $63 \%$ ), mp 190-192 ${ }^{\circ} \mathrm{C}$ (ethanol). IR (KBr): v 2960 (Ali-CH), $1690(\mathrm{C}=\mathrm{O})$, $1620(\mathrm{C}=\mathrm{N}), 1086(\mathrm{C}-\mathrm{O}-\mathrm{C}) \mathrm{cm}^{-1} .{ }^{1} \mathrm{H}-\mathrm{NMR}\left(\mathrm{DMSO}_{6}\right): \delta 2.36\left(\mathrm{~s}, 3 \mathrm{H}, \mathrm{CH}_{3}\right) .{ }^{13} \mathrm{C}-\mathrm{NMR}(\mathrm{DMSO}-$ $\left.\mathrm{d}_{6}\right): \delta 15.83\left(\mathrm{CH}_{3}\right), 142.12$ (C-6, C-7), 150.84, 150.93 (C-4a, C-8a), 154.12 (C-9a), 164.87 (C-2), 170.66, 171.76 (C-5, C-8). MS m/z (\%): 273/269 (M+, 27), 228 (31), 157 (24), 129 (18), 101 (32), 43 (100). Anal. Calcd. for $\mathrm{C}_{9} \mathrm{H}_{3} \mathrm{Cl}_{2} \mathrm{~N}_{3} \mathrm{O}_{3}$ : C, 39.73; H, 1.11; N, 15.17; Cl, 26.06. Found: C, $40.01 ; \mathrm{H}, 1.26 ; \mathrm{N}, 15.28 ; \mathrm{Cl}, 25.79$.

2-Phenyl-6,7-dichloro-benzo[4,5]imidazo[2,1-b][1,3,4]oxadiazole-5,8-dione (6b). Pale green crystals (216 mg, $65 \%)$, mp 210-212 ${ }^{\circ} \mathrm{C}$ (acetonitrile). IR (KBr): v $1695(\mathrm{C}=\mathrm{O}), 1625(\mathrm{C}=\mathrm{N})$, 1600 (aryl), 1085 (C-O-C) $\mathrm{cm}^{-1} .{ }^{1} \mathrm{H}-\mathrm{NMR}\left(\mathrm{DMSO}_{6}\right): \delta$ 7.19-7.66 (m, 5H, aryl). ${ }^{13} \mathrm{C}-\mathrm{NMR}$ (DMSO-d ${ }_{6}$ ): $\delta$ 127.94, 128.81, 129.44 (aryl-CH), 131.22 (aryl-C), 142.26 (C-6, C-7), 150.87, 151.11 (C-4a, C-8a), 154.22 (C-9a), 164.96 (C-2), 170.72, 171,83 (C-5, C-8a). MS m/z (\%): 335/331 (M+, 21), 228 (26), 200 (17), 129 (19), 105 (100), 71 (71). Anal. Calcd. for $\mathrm{C}_{14} \mathrm{H}_{5} \mathrm{Cl}_{2} \mathrm{~N}_{3} \mathrm{O}_{3}$ : C, 50.33; H, 1.51; N, 12.58; Cl, 21.58. Found: C, 50.56; H, 1.32; N, 12.37; Cl, 21.37 .

2-(4-Hydroxyphenyl)-6,7-dichloro-benzo[4,5]imidazo[2,1-b][1,3,4]oxadiazole-5,8-dione (6c). Pale green crystals (234 mg, $67 \%$ ), mp 214-216 ${ }^{\circ} \mathrm{C}$ (acetonitrile). IR (KBr): v $3460(\mathrm{OH}), 1695$ $(\mathrm{C}=\mathrm{O}), 1625(\mathrm{C}=\mathrm{N}), 1595(\operatorname{aryl}), 1088(\mathrm{C}-\mathrm{O}-\mathrm{C}) \mathrm{cm}^{-1} .{ }^{1} \mathrm{H}-\mathrm{NMR}\left(\mathrm{DMSO}_{\mathrm{d}}\right): \delta$ 6.87-7.48 (m, 4H, aryl), 9.46 (br, 1H, OH). ${ }^{13} \mathrm{C}-\mathrm{NMR}$ (DMSO-d6): $\delta$ 126.93, 129.11 (aryl-CH), 130.14, 141.87 (C6, C-7), 151.26, 151.64 (C-4a, C-8a), 154.14 (C-9a), 157.52 (aryl-C), 165.11 (C-2), 170.66, 171.76 (C-5, C-8). MS m/z (\%): 351/347 (M+, 23), 228 (16), 157 (28), 129 (16), 121 (100), 93 (81), 77 (62). Anal. Calcd. for $\mathrm{C}_{14} \mathrm{H}_{5} \mathrm{Cl}_{2} \mathrm{~N}_{3} \mathrm{O}_{4}$ : C, 48.03; H, 1.44; N, 12.00; Cl, 20.25. Found: C, 47.81; H, 1.66; N, 12.28; Cl, 20.47.

2-(4-Bromobenzyl)-6,7-dichloro-benzo[4,5]imidazo[2,1-b][1,3,4]oxadiazole-5,8-dione (6d). Pale green crystals (260 mg, $61 \%$ ), mp 242-246 ${ }^{\circ} \mathrm{C}$ (ethanol). IR (KBr): v 2975 (Ali-CH), 1690 $(\mathrm{C}=\mathrm{O}), 1620(\mathrm{C}=\mathrm{N}), 1600$ (aryl), $1085(\mathrm{C}-\mathrm{O}-\mathrm{C}) \mathrm{cm}^{-1} .{ }^{1} \mathrm{H}-\mathrm{NMR}\left(\mathrm{DMSO}_{6}\right): \delta 4.28\left(\mathrm{~s}, 2 \mathrm{H}, \mathrm{CH}_{2}\right)$, 6.98-7.42 (m, 4H, aryl). ${ }^{13} \mathrm{C}-\mathrm{NMR}$ (DMSO-d6): $\delta 52.64\left(\mathrm{CH}_{2}\right), 126.23$ (aryl-CH), 126.24 (aryl- 
C), 129.88 (aryl-CH), 131.33 (aryl-C), 141.92 (C-6, C-7), 151.11, 151.22 (C-4a, C-8a), 154.33 (C-9a), 164.92 (C-2), 170.74, 171.83 (C-5, C-8). MS m/z (\%): 431/425 (M+2, 28), 327 (22), 229 (31), 198 (63), 118 (27), 91 (66), 77 (100). Anal. Calcd. for $\mathrm{C}_{15} \mathrm{H}_{6} \mathrm{BrCl}_{2} \mathrm{~N}_{3} \mathrm{O}_{3}$ : C, 42.19; $\mathrm{H}, 1.42$; N, 9.84; Cl, 16.60. Found: C, 41.92; H, 1.31; N, 10.05; Cl, 16.83 .

2,3,7,8-Tetrachlorothianthrene-1,4,6,9-tetraone (7). Yield (with 1a, $41 \mathrm{mg}$ (10\%); 1b, $50 \mathrm{mg}$ (12\%); 1c, $58 \mathrm{mg}(14 \%)$; 1d, $37 \mathrm{mg}(9 \%) \mathrm{mp} 342-344{ }^{\circ} \mathrm{C}\left(\mathrm{lit}^{20} .342-344{ }^{\circ} \mathrm{C}\right)$

\section{Reaction of 1-acylthiosemicarbazides 1a-d with 3a}

A mixture of equimolar amounts of the appropriate 1-acylthiosemicarbazide 1a-d and 3a was stirred under reflux in $30 \mathrm{ml}$ of dry ethyl acetate for 3 hours. The mixture was concentrated under vacuum and the residue separated by plc using cyclohexane / ethyl acetate (1:1) as developing solvent to give numerous coloured zones, two of which (with the highest intensity) were extracted and removed. The fastest migrating one $\mathrm{R}_{\mathrm{f}}=0.192$ contained naphthimidazoxadiazoledione 11a-d, the second zone $R_{f}=0.144$ (which was always characterized by a blue colour) contained the naphthobisthiazole derivatives 12a-d. Extraction of the zones with acetone, and concentration gave a residue, which was rechromatographed to separate the pure compounds.

2-Methylnaphtho[4,5]imidazo[2,1-b][1,3,4]oxadiazole-5,10-dione (11a). Reddish brown crystals $(76 \mathrm{mg}, 30 \%), \mathrm{mp} 183-185^{\circ} \mathrm{C}$ (acetonitrile). IR $(\mathrm{KBr}): v 1670(\mathrm{C}=\mathrm{O}), 1625(\mathrm{C}=\mathrm{N})$, 1590 (aryl), $1085(\mathrm{C}-\mathrm{O}-\mathrm{C}) \mathrm{cm}^{-1} .{ }^{1} \mathrm{H}-\mathrm{NMR}\left(\mathrm{DMSO}_{6}\right): \delta 2.35\left(\mathrm{~s}, 3 \mathrm{H}, \mathrm{CH}_{3}\right), 7.24-7.86(\mathrm{~m}, 4 \mathrm{H}$, aryl). ${ }^{13} \mathrm{C}-\mathrm{NMR}\left(\mathrm{DMSO}_{6}\right): \delta 15.83\left(\mathrm{CH}_{3}\right), 128.66,129.89$ (aryl-CH), 132.14 (aryl-C), 150.87, 151.26 (C-4a, C-10a), 154.14 (C-11a), 164.63 (C-2), 173.38 (C-5, C-10). MS m/z (\%): 253 (M , 28), 210 (19), 182 (21), 154 (11), 132 (36), 105 (67), 76 (54), 43 (100). Anal. Calcd. for $\mathrm{C}_{13} \mathrm{H}_{7} \mathrm{~N}_{3} \mathrm{O}_{3}$ : C, 61.66; H, 2.79; N, 16.59. Found: C, 61.87; H, 2.61; N, 16.32 .

2-Phenylnaphtho[4,5]imidazo[2,1-b][1,3,4]oxadiazole-5,10-dione (11b). Reddish brown crystals $(104 \mathrm{mg}, 33 \%), \mathrm{mp} 222-234{ }^{\circ} \mathrm{C}$ (methanol). IR $(\mathrm{KBr}): v 1690(\mathrm{C}=\mathrm{O}), 1620(\mathrm{C}=\mathrm{N})$, 1600 (aryl), 1080 (C-O-C) $\mathrm{cm}^{-1} .{ }^{1} \mathrm{H}-\mathrm{NMR}\left(\mathrm{DMSO}_{6}\right): \delta 7.22-7.89$ (m, 9H, aryl). ${ }^{13} \mathrm{C}-\mathrm{NMR}$ (DMSO-d $)_{\text {) }} \delta$ 127.96, 128.53, 128.84, 129.33, 129.84 (aryl-CH), 132.16, 132.88 (aryl-C), 150.76, 151.14 (C-4a, C-10a), 154.33 (C-11a), 164.42 (C-2), 173.36 (C-5, C-10). MS m/z (\%): $315\left(\mathrm{M}^{+}, 21\right), 210$ (18), 182 (14), 154 (10), 132 (22), 105 (100), 77 (64), 65 (52). Anal. Calcd. for $\mathrm{C}_{18} \mathrm{H}_{9} \mathrm{~N}_{3} \mathrm{O}_{3}: \mathrm{C}, 68.57 ; \mathrm{H}, 2.88 ; \mathrm{N}, 13.33$. Found: C, 68.81; H, 3.04; N, 13.05.

2-(4-Hydroxyphenyl)naphtho[4,5]imidazo[2,1-b][1,3,4]oxadiazole-5,10-dione (11c). Reddish brown crystals (106 mg, $32 \%$ ), mp 259-261 ${ }^{\circ} \mathrm{C}$ (acetonitrile). IR (KBr): $v 3460(\mathrm{OH}), 1695$ $(\mathrm{C}=\mathrm{O}), 1625(\mathrm{C}=\mathrm{N}), 1590$ (aryl), $1085(\mathrm{C}-\mathrm{O}-\mathrm{C}) \mathrm{cm}^{-1} .{ }^{1} \mathrm{H}-\mathrm{NMR}\left(\mathrm{DMSO}_{6}\right): \delta$ 6.98-7.73 (m, 8H, aryl), 9.09 (br, 1H, OH). ${ }^{13} \mathrm{C}-\mathrm{NMR}\left(\mathrm{DMSO}_{\mathrm{d}}\right.$ ): $\delta$ 126.72, 128.66, 128.92, 129.87 (aryl-CH), 131.12, 132.88 (aryl-C), 150.86, 151.33 (C-4a, C-10a), 154.14 (C-11a), 156.12 (aryl-C), 164.52 (C-2), 173.47 (C-5, C-10). MS m/z (\%): 331 (M+32), 210 (18), 154 (22), 121 (86), 105 (93), 92 (86), 77 (100), 65 (63). Anal. Calcd. for $\mathrm{C}_{18} \mathrm{H}_{9} \mathrm{~N}_{3} \mathrm{O}_{4}$ : C, 65.26; H, 2.74; N, 12.68. Found: C, 64.97; H, 2.91; N, 12.53 . 
2-(4-Bromobenzyl)naphtho[4,5]imidazo[2,1-b][1,3,4]oxadiazole-5,10-dione (11d). Reddish brown crystals $\left(111 \mathrm{mg}, 28 \%\right.$ ), mp 195-197 ${ }^{\circ} \mathrm{C}$ (methanol). IR (KBr): v 2975 (Ali-CH), 1690 $(\mathrm{C}=\mathrm{O}), 1625(\mathrm{C}=\mathrm{N}), 1600$ (aryl), $1080(\mathrm{C}-\mathrm{O}-\mathrm{C}) \mathrm{cm}^{-1} .{ }^{1} \mathrm{H}-\mathrm{NMR}\left(\mathrm{DMSO}_{6} \mathrm{~d}_{6}\right): \delta 4.24\left(\mathrm{~s}, 2 \mathrm{H}, \mathrm{CH}_{2}\right)$, 7.12-7.82 (m, 8H, aryl). ${ }^{13} \mathrm{C}-\mathrm{NMR}\left(\mathrm{DMSO}_{\mathrm{d}}\right): \delta 52.57\left(\mathrm{CH}_{2}\right), 126.88$ (aryl-C), 128.57, 129.88, 130.67, 131.12 (aryl-CH), 132.94, 135.37 (aryl-C), 151.27 (C-4a, C-10a), 152.96 (C-11a), 165.12 (C-2), 173.56 (C-5, C-10). MS m/z (\%): 394/396 (M+3 , 31), 314 (22), 198 (57), 196 (36), 168 (16), 140 (9), 105 (61), 91 (72), 77 (100), 65 (48). Anal. Calcd. for $\mathrm{C}_{19} \mathrm{H}_{10} \mathrm{BrN}_{3} \mathrm{O}_{3}: \mathrm{C}, 54.71$; H, 2.30; N, 10.63. Found: C, 54.96; H, 2.46; N, 10.39.

Naphtho[1,2-d:4,3- $d$ ]bis(imidazo[2,1-b][1,3,4]oxadiazole)acetylhydrazide $\quad$ (12a). Blue crystals (212 mg, $55 \%$ ), mp 200-202 ${ }^{\circ} \mathrm{C}$ (acetonitrile). IR (KBr): v 3390, $3215(\mathrm{NH}), 1670$ $(\mathrm{C}=\mathrm{O}), 1600(\operatorname{aryl}) \mathrm{cm}^{-1} .{ }^{1} \mathrm{H}-\mathrm{NMR}\left(\mathrm{DMSO}_{6}\right): \delta 2.36\left(\mathrm{~s}, 6 \mathrm{H}, \mathrm{CH}_{3}\right), 7.10-7.48(\mathrm{~m}, 4 \mathrm{H}$, aryl), 10.64 (br, 2H, thiazole-NH), 11.12 (br, 2H, amide-NH). ${ }^{13} \mathrm{C}-\mathrm{NMR}$ (DMSO-d $)$ : $\delta 21.12\left(\mathrm{CH}_{3}\right)$, 127.93, 128.27 (aryl-CH), 130.26, 134.61, 135.74 (aryl-C), 162.66 (C-2), 171.12 (amide-CO). MS m/z (\%): $386\left(\mathrm{M}^{+}, 19\right), 343$ (16), 300 (24), 244 (31), 164 (21), 120 (11), 77 (54), 43 (100). Anal. Calcd. for $\mathrm{C}_{16} \mathrm{H}_{14} \mathrm{~N}_{6} \mathrm{O}_{2} \mathrm{~S}_{2}$ : C, 49.73; H, 3.65; N, 21.75; S, 16.59. Found: C, 49.51; H, 3.81; N, 21.96; S, 16.37.

Naphtho[1,2-d:4,3-d $]$ bis(imidazo[2,1-b][1,3,4] oxadiazole)benzohydrazide $\quad(12 b) . \quad$ Blue crystals (301 mg, $59 \%$ ), mp 285-287 ${ }^{\circ} \mathrm{C}$ (acetonitrile). IR (KBr): v 3385, $3220(\mathrm{NH}), 1675$ $(\mathrm{C}=\mathrm{O}), 1610$ (aryl) $\mathrm{cm}^{-1} .{ }^{1} \mathrm{H}-\mathrm{NMR}\left(\mathrm{DMSO}_{-} \mathrm{d}_{6}\right): \delta$ 7.16-7.98 (m, 14H, aryl), 10.52 (br, 2H, thiazole-NH), 11.16 (br, 2H, amide-NH). ${ }^{13} \mathrm{C}-\mathrm{NMR}$ (DMSO-d $\left.\mathrm{D}_{6}\right): \delta 127.84,127.93,128.96$, 130.36 (aryl-CH), 130.78, 134.66, 135.76 (aryl-C), 163.11 (C-2), 171.37 (amide-CO). MS m/z (\%): $510\left(\mathrm{M}^{+}, 25\right), 405$ (14), 300 (19), 244 (23), 164 (12), 105 (100), 77 (53). Anal. Calcd. for $\mathrm{C}_{26} \mathrm{H}_{18} \mathrm{~N}_{6} \mathrm{O}_{2} \mathrm{~S}_{2}$ : C, 61.16; H, 3.55; N, 16.46; S, 12.56. Found: C, 60.93; H, 3.74; N, 16.71; S, 12.32 .

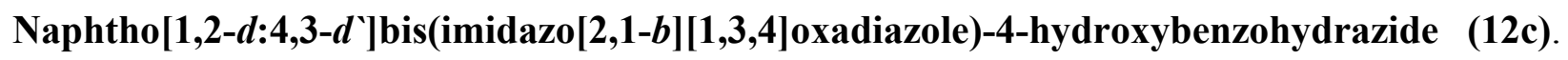
Blue crystals (309 mg, 57 \%), mp 262-264 ${ }^{\circ} \mathrm{C}$ (acetonitrile). IR (KBr): v 3370, $3240(\mathrm{OH}, \mathrm{NH})$, $1670(\mathrm{C}=\mathrm{O}), 1600$ (aryl) $\mathrm{cm}^{-1} .{ }^{1} \mathrm{H}-\mathrm{NMR}\left(\mathrm{DMSO}_{\mathrm{d}}\right): \delta 6.98-7.88(\mathrm{~m}, 12 \mathrm{H}$, aryl), $9.58(\mathrm{br}, 1 \mathrm{H}$, $\mathrm{OH}), 10.63$ (br, 2H, thiazole-NH), 11.22 (br, 2H, amide-NH). MS m/z (\%): $542\left(\mathrm{M}^{+}, 18\right), 421$ (19), 300 (9), 244 (17), 188 (13), 121 (71), 120 (100), 92 (82), 77 (63).. Anal. Calcd. for $\mathrm{C}_{26} \mathrm{H}_{18} \mathrm{~N}_{6} \mathrm{O}_{4} \mathrm{~S}_{2}$ : C, 57.55; H, 3.34; N, 15.49; S, 11.82. Found: C, 57.77; H, 3.19; N, 15.27; S, 12.08 .

Naphtho[1,2-d:4,3-d $]$ bis(imidazo[2,1-b][1,3,4]oxadiazole)-2-(4-bromophenyl)acetohydrazide (12d). Blue crystals (368 mg, $53 \%$ ), mp 214-216 ${ }^{\circ} \mathrm{C}$ (acetonitrile). IR (KBr): $v 3385$, $3225(\mathrm{NH}), 1670(\mathrm{C}=\mathrm{O}), 1595$ (aryl) $\mathrm{cm}^{-1} .{ }^{1} \mathrm{H}-\mathrm{NMR}\left(\mathrm{DMSO}-\mathrm{d}_{6}\right): \delta 4.28\left(\mathrm{~s}, 4 \mathrm{H}, \mathrm{CH}_{2}\right), 6.98-7.42$ (m, 12H, aryl), 10.65 (br, 2H, thiazole-NH), 11.17 (br, 2H, amide-NH). ${ }^{13} \mathrm{C}-\mathrm{NMR}$ (DMSO-d 6 ): $\delta$ $52.28\left(\mathrm{CH}_{2}\right), 128.86,129.82,130.96,131.22$ (aryl-CH), 132.12, 134.76 (aryl-C), 163.26 (C-2), 171.48 (amide-CO). MS m/z (\%): 698/694 (M+, 17), 524 (18), 298 (24), 198 (36), 118 (24), 91 (38), 77 (100), 65 (64). Anal. Calcd. for $\mathrm{C}_{28} \mathrm{H}_{20} \mathrm{Br}_{2} \mathrm{~N}_{6} \mathrm{O}_{2} \mathrm{~S}_{2}$ : C, 48.29; H, 2.89; N, 12.07; S, 9.21. Found: C, 48.41; H, 3.11; N, 11.82; S, 9.47. 


\section{Reaction of 1-acylthiosemicarbazides 1a-d with (3b)}

A solution of 1a-d (1 mmol) in $20 \mathrm{ml}$ of dry ethyl acetate is added dropwise to solution of $\mathbf{3 b}$ (1 $\mathrm{mmol}$ ) in $10 \mathrm{ml}$ of dry ethyl acetate at room temperature. The reaction mixture becomes green and gradually turns into a reddish brown colour. It was left standing for 48 hours, concentrated in vacuo and the residue was subjected to plc using cyclohexane/ethyl acetate (2:1) to give numerous coloured zones, the two intense of which were removed and extracted. The fastest migrating zone which quenched all indicator fluorescence upon exposure to $254 \mathrm{~nm}$ UV-light contained oxadiazole derivatives 4a-d and the slowest migrating zone (which is always characterized by orange colour) contained the naphthothiazine derivatives 13a-d. Extraction of the zones with acetone and recrystallization afforded the reaction products.

\section{(4-Amino-5-cyano-6-hydroxy-2H-naphtho[2,1-e][1,3] thiazine-2-ylidene)acetohydrazide}

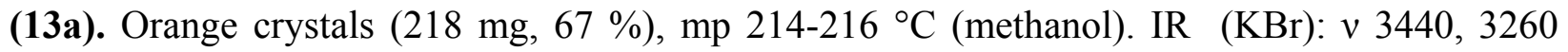
$\left(\mathrm{OH}, \mathrm{NH}, \mathrm{NH}_{2}\right), 2215(\mathrm{CN}), 1670(\mathrm{C}=\mathrm{O}), 1625(\mathrm{C}=\mathrm{N}), 1595$ (aryl) $\mathrm{cm}^{-1} .{ }^{1} \mathrm{H}-\mathrm{NMR}\left(\mathrm{DMSO}-\mathrm{d}_{6}\right)$ : $\delta 2.37\left(\mathrm{~s}, 3 \mathrm{H}, \mathrm{CH}_{3}\right), 7.16\left(\mathrm{br}, 2 \mathrm{H}, \mathrm{NH}_{2}\right), 7.35-7.84(\mathrm{~m}, 4 \mathrm{H}$, aryl), 9.62 (br, $1 \mathrm{H}, \mathrm{OH}), 11.18$ (br, $1 \mathrm{H}$, amide-NH). ${ }^{13} \mathrm{C}-\mathrm{NMR}\left(\mathrm{DMSO}_{\mathrm{d}}\right): \delta 22.34\left(\mathrm{CH}_{3}\right), 117.84(\mathrm{CN}), 126.74,127.82,128.35$, 129.88 (aryl-CH), 132.14,136.67 (aryl-C), 153.82 (C-6), 154.93 (C-2), 156.76 (C-4), 171.42 (amide-CO). MS m/z (\%): $325\left(\mathrm{M}^{+}, 31\right), 282$ (24), 254 (18), 238 (11), 175 (19), 76 (32), 43 (100). Anal. Calcd. for $\mathrm{C}_{15} \mathrm{H}_{11} \mathrm{~N}_{5} \mathrm{O}_{2} \mathrm{~S}$ : C, 55.38; H, 3.41; N, 21.53; S, 9.86. Found: C, 55.59; H, $3.63 ; \mathrm{N}, 21.29 ; \mathrm{S}, 10.05$.

\section{(4-Amino-5-cyano-6-hydroxy-2H-naphtho[2,1-e][1,3]thiazine-2-ylidene)benzohydrazide}

(13b). Orange crystals (279 mg, $72 \%$ ), mp 228-230 ${ }^{\circ} \mathrm{C}$ (acetonitrile). IR (KBr): v 3445, 3370$3250\left(\mathrm{OH}, \mathrm{NH}, \mathrm{NH}_{2}\right), 2220(\mathrm{CN}), 1675(\mathrm{C}=\mathrm{O}), 1620(\mathrm{C}=\mathrm{N}), 1600$ (aryl) cm ${ }^{-1} .{ }^{1} \mathrm{H}-\mathrm{NMR}$ $\left(\mathrm{DMSO}_{\mathrm{d}}\right.$ ): $\delta 7.12$ (br, 2H, $\mathrm{NH}_{2}$ ), 7.31-7.95 (m, 9H, aryl), 9.57 (br, 1H, OH), 11.15 (br, 1H, amide-NH). ${ }^{13} \mathrm{C}-\mathrm{NMR}$ (DMSO-d $\left.{ }_{6}\right): \delta 118.11(\mathrm{CN}), 126.58,127.12,127.54,127.76,128.24$, 128.93, 129.86 (aryl-CH), 130.86, 132.38, 134.76 (aryl-C), 153.65 (C-6), 154.84 (C-2), 156.93 (C-4), 171.56 (amide-CO). MS m/z (\%): 387 (M $\left.\mathrm{M}^{+}, 23\right), 282$ (19), 240 (24), 175 (12), 105 (100), 77 (53), 65 (41). Anal. Calcd. for $\mathrm{C}_{20} \mathrm{H}_{13} \mathrm{~N}_{5} \mathrm{O}_{2} \mathrm{~S}$ : C, 62.00; H, 3.38; N, 18.08; S, 8.28. Found: C, $61.78 ; \mathrm{H}, 3.56$; N, 17.89; S, 8.05.

(4-Amino-5-cyano-6-hydroxy-2H-naphtho[2,1-e][1,3] thiazine-2-ylidene)-4-hydroxybenzohydrazide (13c). Reddish orange crystals (278 $\mathrm{mg}, 69 \%$, mp 233-235 ${ }^{\circ} \mathrm{C}$ (acetonitrile). IR $(\mathrm{KBr}):$ v 3470, 3380-3260 (OH, NH, $\left.\mathrm{NH}_{2}\right), 2220(\mathrm{CN}), 1670(\mathrm{C}=\mathrm{O}), 1620(\mathrm{C}=\mathrm{N}), 1595$ (aryl) $\mathrm{cm}^{-1} .{ }^{1} \mathrm{H}-\mathrm{NMR}\left(\mathrm{DMSO}_{6}\right): \delta$ 7.05-7.82 (m, 10H, $\mathrm{NH}_{2}$ and aryl), 9.57 (br, 1H, OH), 9.68 (br, 1H, $\mathrm{OH}), 11.18$ (br, 1H, amide-NH). MS m/z (\%): 403 (M+, 34), 282 (16), 254 (12), 211 (6), 175 (8), 121 (67), 104 (83), 77 (100), 65 (56). Anal. Calcd. for $\mathrm{C}_{20} \mathrm{H}_{13} \mathrm{~N}_{5} \mathrm{O}_{3} \mathrm{~S}$ : C, 59.55; H, 3.25; N, 17.36; S, 7.95. Found: C, 59.31; H, 3.48; N, 17.54; S, 8.19.

\section{(4-Amino-5-cyano-6-hydroxy-2H-naphtho[2,1-e][1,3]thiazine-2-ylidene)-2-(4-bromo-}

phenyl)acetohydrazide (13d). Orange crystals (312 mg, $65 \%$ ), mp 248-250 ${ }^{\circ} \mathrm{C}$ (methanol). IR $(\mathrm{KBr}): v$ 3430, 3380-3260 (OH, NH, $\left.\mathrm{NH}_{2}\right), 2220(\mathrm{CN}), 1675(\mathrm{C}=\mathrm{O}), 1615(\mathrm{C}=\mathrm{N}), 1590$ (aryl) $\mathrm{cm}^{-1} .{ }^{1} \mathrm{H}-\mathrm{NMR}\left(\mathrm{DMSO}_{6}\right): \delta 4.26\left(\mathrm{~s}, 2 \mathrm{H}, \mathrm{CH}_{2}\right), 7.05-7.78\left(\mathrm{~m}, 10 \mathrm{H}, \mathrm{NH}_{2}\right.$ and aryl), $9.65(\mathrm{br}, 1 \mathrm{H}$, $\mathrm{OH}), 11.16$ (br, 1H, amide-NH). ${ }^{13} \mathrm{C}-\mathrm{NMR}\left(\mathrm{DMSO}_{\mathrm{d}}\right): \delta 52.61\left(\mathrm{CH}_{2}\right), 126.94,127.73,128.26$, 
129.85, 130.12, 130.63 (aryl-CH), 131.22, 132.36, 134.75 (aryl-C), 153.58 (C-6), 154.68 (C-2), 156.76 (C-4), 171.48 (amide-CO). MS m/z (\%): 481/479 ( $\left.\mathrm{M}^{+}, 27\right), 400$ (12), 282 (19), 254 (6), 228 (7), 198 (28), 118 (68), 77 (100), 65 (74). Anal. Calcd. for $\mathrm{C}_{21} \mathrm{H}_{14} \mathrm{BrN}_{5} \mathrm{O}_{2} \mathrm{~S}$ : C, 52.51; $\mathrm{H}$, 2.94; N, 14.58; S, 6.68. Found: C, 52.24; H, 3.98; N, 14.76; S, 6.41.

\section{Acknowledgements}

A. A. Hassan is indepted to the A. v. Humbodlt- Foundation for the donation of the Shimadzu 408 IR spectrophotometer.

\section{References}

1. Patai, S.; Rappoport, Z. The Chemistry of Quinonoid Compounds; Wiley Interscience : New York, 1988; Vol. 2. , Part 1, pp 552-570

2. Ulrich, H.; Richter, R. In Methoden der Organischen Chemie, Chinone Teil 1, p-Chinone der Benzol und Naphthalin Reihen, C. Grundmann, Ed., Georg-Thieme Verlag: Stuttgart 1977.

3. Lee, H-J.; Park, S-Y.; Kim, J. S.; Song, H. M.; Suh, M-E.; Lee, C-O. Bioorg. Med, Chem. 2003, 11, 4791.

4. Vanelle, P.; Donini, S.; Maldonado, J.; Crozet, M. P.; Delmas, F.; Gasquet, M.; David, P. T-. Eur. J. Med. Chem. 1997, 32, 523.

5. Monterrey, I. G-; Campiglia, P.; Grieco, P.; Diurno, M. V.; Bolognese, A.; Colla, P. L.; Novellino, E. Bioorg. Med. Chem. 2003, 11, 3769.

6. Lee, H-J.; Suh, M-E.; Lee, C-O. Bioorg. Med. Chem. 2003, 11, 1511.

7. Bittner, S.; Temtsin, G.; Sasson, Y. Synthesis 2000, 1084.

8. Dawadi, P. B. S-.; Bittner, S.; Fridkin, M.; Rahimipiour, S. Synthesis 1996, 1468.

9. Ballesteros, P.; Claramunt, R. M.; Escolastico, C.; Santa Maria, M. D. J. Org. Chem. 1993, $57,1873$.

10. Hassan, A. A.; Ibrahim, Y. R.; Semida, A. A.; Mourad, A. E. Liebigs Ann. Chem. 1994, 989.

11. Hassan, A. A.; Mohamed, N. K.; Ibrahim, Y. R.; Sadek, K. U.; Mourad, A. E. Bull. Chem. Soc. Jpn. 1993, 66, 2612.

12. Katritzky, A. R.; Fan, W. Q. J. Heterocyclic Chem. 1988, 25, 901.

13. Katritzky, A. R.; Fan, W. Q.; Linand, Q. I.; Bayyuk, S. J. Heterocyclic Chem. 1989, $26,885$.

14. Matsuoka, M.; Iwamoto, A.; Furukawa, N.; Kitao, T. J. Heterocyclic Chem. 1992, 29, 439.

15. Matsuoka, M.; Iwamoto, A. J. Heterocyclic Chem. 1993, 30, 173.

16. Katritzky, A. R.; Fan, W. Q. J. Heterocyclic Chem. 1993, 30, 1679. 
17. Nour El-Din, A. M.; Mourad, A. E.; Hassan, A. A.; Gomaa, M. A. Bul. Chem. Soc. Jpn. 1991, 64, 1966.

18. Döpp, D.; Gomaa, M. A.; Henkel, G.; Nour El-Din, A. M. J. Heterocyclic Chem. 1995, 32, 603.

19. Ali, A. A.; Ahmed, E. K.; El-Mokadem, K. M. J. Sulfur. Chem. 2006, 27, 419.

20. Hassan, A. A.; Mourad, A. E.; El-Shaieb, K. M.; Abou-Zied, A. H. J. Heterocyclic Chem. 2006, 43, 471.

21. Hassan, A. A.; Ibrahim, Y. R.; Shawky, A. M. J. Sulfur Chem. 2007, 28, 211.

22. Kalinowski, H. O.; Berger, S.; Braun, S. ${ }^{13} C-N M R$ Spectroscopy; Georg Thieme Verlag: Stuttgart, 1984.

23. Silverstein, R. M. Spectrometric Identification of Organic Compounds; John Wiley and Sons, Inc.: New York, 1974.

24. Foster, R.; Horman, I. J. Chem. Soc. B. 1966, 1049.

25. Beyer, H.; Hohn, H.; Lossig, W. Chem. Ber. 1952, 85, 1122.

26. Wang, Z.; Shi, H. Synthetic Comm. 2001, 31, 2841.

27. Hogyrath, E. J. Chem. Soc. 1949, 1167.

28. Budni, M. L.; Jayadevappa, E. S. Spectrochim. Acta, Part A 1988, 44, 607.

29. De, S. C. J. Indian Chem. Scoc. 1930, 70, 651.

30. Henkleiss, P.; Westphal, G.; Kraft, R. Tetrahedron 1973, 29, 2937.

31. R. K. Bansal, J. Indian Chem. Soc. 1982, 59, 277. 